\title{
Modélisation d'écoulements pulsés de fluides diphasiques en conduites viscoélastiques
}

\author{
Two-fluid pulsatile flows modelisation through viscoelastic ducts
}

\author{
par H. Amar, M. Kerroum, Zeggwagh
}

Groupe de Mécanique des Fluides et des Structures. Laboratoire de Mécanique, Faculté des Sciences de Rabat

The present work deals with a two-fluid model for blood through deformable tubes of small diameter. This two-fluid model consists of a core (suspension of red cells) and peripheral red cell free plasma layer. The core has been considered as ostwaldian fluid and the plasma layer as a newtonian fluid. Using an implicit difference method to resolve the equations governing such flows, we determine the axial velocity profiles. This study, considered as a step in modelling of flow in blood vessels, may also contribute to other important fields such as water desalination or gel filtration.

\section{INTRODUCTION}

Les écoulements de fluides en conduites, modélisant les écoulements sanguins, ont été étudiés jusqu'à présent avec des hypothèses très diverses.

Ces dernières années ont été marquées par un regain d'intérêt pour l'étude des écoulements en présence d'une couche plasmatique périphérique [1, 2, 3, 4]. Divers modèles ont été utilisés, dans la plupart des cas en géométrie cylindrique, parfois en présence de singularité $[2,5,6]$. La plupart des auteurs $[7,8,9,10]$ ont essayé d'expliquer certains phénomènes associés aux écoulements sanguins en proposant différents modèles théoriques. Haynes [11], pour expliquer l'effet Fahreus-Linqvist, a considéré un modèle diphasique où les deux phases fluides sont newtoniennes, avec des viscosités différentes. Bugliarello et Sevilla [8] ont considéré dans une première étape un modèle dans lequel les deux fluides sont newtoniens avec des viscosités différentes, puis dans une deuxième étape, deux fluides régis par la loi de Casson dans laquelle les contraintes seuil et les viscosités sont différentes. Mais la plupart des travaux expérimentaux [8], [18], [19], [20] montrent que l'écoulement sanguin à travers les petits conduits est diphasique : une phase fluide claire, le plasma, près de la paroi et un noyau constitué de globules rouges [21], [22]. Goldsmith et al. [12], entre autres, ont montré que le plasma se comporte comme un fluide newtonien, tandis que le noyau est non newtonien. Tenant compte des résultats expérimentaux de différents auteurs, Chaturani et Kalani [7] proposent un modèle dipha- sique avec une couche périphérique newtonienne et un noyau modélisé par un fluide à couple de contraintes, en conduite rigide, qui tient compte des effets de cisaillement. Zeggwagh en 1988 [3] a étudié un modèle diphasique Newton-Quemada, en conduite tronconique poreuse et rigide, Kerroum en 1994 [4] traite le modèle binewtonien en conduite cylindrique uniforme viscoélastique.

Compte tenu de ces diverses études et afin de compléter le champ d'investigation de façon à se rapprocher de la réalité hémodynamique, on considère dans cette étude à la fois le caractère non permanent, pulsé, de l'écoulement, l'aspect diphasique du fluide, couche périphérique newtonienne et phase centrale obéissant à la loi d'Ostwald, et la déformabilité de la paroi considérée viscoélastique.

La résolution des systèmes des équations régissant de tels écoulements nécessite, lorsque le gradient de pression est inconnu le long du conduit, ce qui est généralement le cas, la mise en ouvre d'une méthode itérative $[13,14,4]$. Nous utilisons pour cela une méthode numérique aux différences finies implicite et nous étudions l'influence des paramètres rhéologiques du fluide et de la paroi sur l'évolution des profils des vitesses axiales.

\section{II — MODÈLE THÉORIQUE}

La conduite concernée axisymétrique d'axe $\overrightarrow{o z}$, de longueur $L$ de rayons $R_{0}$ et $R^{*}\left(z^{*}, t^{*}\right)$ respectivement au repos et à l'état déformé, est supposée à paroi mince et non poreuse. 
Le comportement rhéologique de la paroi de cette conduite est décrit à tout instant et en chaque section par la loi [15] :

$$
\lambda * \frac{\partial S^{*}}{\partial t^{*}}+S^{*}-S_{0}^{*}=a^{*}\left(P^{*}-P_{e x t}^{*}\right)
$$

où $S^{*}\left(z^{*}, t^{*}\right)$ et $S_{0}^{*}$ sont respectivement la section à l'instant $t$ et à l'état de repos.

$P^{*}$ et $P_{e x t}^{*}$ sont respectivement la pression à l'intérieur et à l'extérieur du tube, cette dernière est supposée constante.

$\lambda$ * est le temps de relaxation lié à la viscoélasticité de la paroi et $a^{*}$ la compliance caractérisant sa rigidité.

L'écoulement, de vitesse axiale $W^{*}\left(r^{*}, z^{*}, t^{*}\right)$ et radiale $U^{*}\left(r^{*}, z^{*}, t^{*}\right)$ en chaque point de l'écoulement $M\left(r^{*}, z^{*}\right)$ supposé à chaque instant à deux phases, est composé d'un noyau central de rayon $A^{*}\left(z^{*}, t^{*}\right)$ de viscosité ostwaldienne :

$$
\eta_{m}^{*}=K^{*} s^{* \frac{n-1}{2}} \text { avec } s^{*}=2 T r d^{*^{2}}
$$

où $K^{*}, n$, et $\mathbf{d}^{*}$ sont respectivement la consistance, l'indice de comportement du fluide et le tenseur des taux de déformations, et d'un manchon périphérique, le plasma, dont le comportement newtonien a pour viscosité dynamique constante $\eta_{p}^{*}$

Des macrophotographies de ce type d'écoulement réalisées par vidéomicroscopie [2], montrent que l'interface entre les deux fluides considérés a le même profil que celui de la paroi du conduit et permet de mesurer l'épaisseur relative du noyau. On posera à tout instant et en chaque section :

$$
A^{*}\left(z^{*}, t^{*}\right)=\alpha R^{*}\left(z^{*}, t^{*}\right)
$$

où $\alpha$ est une constante caractérisant l'épaisseur de la couche plasmatique telle que $0<\alpha<1$.

Cette épaisseur est évaluée dans la littérature à environ un dixième du rayon du vaisseau.

Les équations régissant l'écoulement, en l'absence des efforts volumiques, sont celles traduisant la conservation de la quantité de mouvement et la conservation de la masse.

$$
\rho \frac{d V^{*}}{d t^{*}}=\widetilde{\operatorname{div} \Sigma^{*}}
$$

et $\operatorname{div} \overrightarrow{V^{*}}=0$ avec $\Sigma^{*}=-P^{*} \boldsymbol{I}+2 \eta^{*} \boldsymbol{d}^{*}$

où $\overrightarrow{V^{*}}$ est le champ de vitesses recherché sous la forme:

$$
\overrightarrow{V^{*}}\left(r^{*}, z^{*}, t^{*}\right)=U^{*}\left(r^{*}, z^{*}, t^{*}\right) \overrightarrow{e_{r}^{*}}+W^{*}\left(r^{*}, z^{*}, t^{*}\right) \overrightarrow{e_{z}^{*}}
$$

$\overrightarrow{\Sigma^{*}}$ est le tenseur de contraintes et $\mathbf{I}$ le tenseur identité.

On associe à ce système d'équations les conditions aux limites traduisant l'axisymétrie de l'écoulement et l'adhérence à la paroi.

A l'interface on impose la continuité du champ des vitesses et des contraintes tangentielles.

\section{III —ADIMENSIONNALISATION ET SIM- PLIFICATION DES ÉQUATIONS}

Dans le but de mettre en évidence des nombres sans dimension, caractéristiques de l'écoulement, on définit les quantités adimensionnelles suivantes :

$$
\begin{aligned}
& z=\frac{z^{*}}{L}, r=\frac{r^{*}}{R_{0}}, t=\frac{t^{*} \omega}{2 \pi}, W=\frac{W^{*}}{W_{0}}, U=\frac{U^{*} L}{R_{0} W_{0}}, P=\frac{P^{*} R_{0}}{\eta_{p} L W_{0}} \\
& \text { et } \eta=\frac{\eta^{*}}{\eta_{p}}
\end{aligned}
$$

$W_{0}$ est une vitesse caractéristique de l'écoulement dans la direction axiale, $\omega$ la pulsation du phénomène et $P$ la pression.

Les grandeurs en * sont les grandeurs dimensionnelles.

Les équations adimensionnelles s'écrivent alors :

$$
\begin{aligned}
& \beta^{2} \varepsilon^{2} \frac{\partial U}{\partial t}+\operatorname{Re} \varepsilon^{3}\left(U \frac{\partial U}{\partial r}+W \frac{\partial U}{\partial z}\right)=-\frac{\partial P}{\partial r}+2 \varepsilon^{2} \eta \frac{\partial^{2} U}{\partial r^{2}}+2 \varepsilon^{2} \frac{\partial U \partial \eta}{\partial r} \partial r \\
& +\varepsilon^{2} \eta \frac{\partial}{\partial z}\left(\varepsilon^{2} \frac{\partial U}{\partial z}+\frac{\partial W}{\partial r}\right)+\varepsilon^{2}\left(\varepsilon^{2} \frac{\partial U}{\partial z}+\frac{\partial W}{\partial r}\right) \frac{\partial \eta}{\partial z}+2 \varepsilon^{2} \eta \frac{\partial U}{\partial r}-2 \varepsilon^{2} \eta \frac{U}{r^{2}} \\
& \beta^{2} \frac{\partial W}{\partial t}+\operatorname{Re} \varepsilon\left(W \frac{\partial W}{\partial z}+U \frac{\partial W}{\partial r}\right)=-\frac{\partial P}{\partial z}+\eta \frac{\partial}{\partial r}\left(\varepsilon^{2} \frac{\partial U}{\partial z}+\frac{\partial W}{\partial r}\right) \\
& \quad+\left(\varepsilon^{2} \frac{\partial U}{\partial z}+\frac{\partial W}{\partial r}\right) \frac{\partial \eta}{\partial r}+2 \varepsilon^{2} \eta \frac{\partial^{2} W}{\partial z^{2}}+2 \varepsilon^{2} \frac{\partial W \partial \eta}{\partial z}+\frac{\eta}{r z}\left(\varepsilon^{2} \frac{\partial U}{\partial z}+\frac{\partial W}{\partial r}\right) \\
& \frac{\partial W}{\partial z}+\frac{1}{r} \frac{\partial(r U)}{\partial r}=0
\end{aligned}
$$

Cette adimensionnalisation, où la viscosité du manchon a été prise comme viscosité de référence et où les termes de pression sont pris du même ordre de grandeur que ceux de viscosité, introduit dans les équations les nombres sans dimension, caractéristiques de l'écoulement, suivants :

$$
\beta=R_{0} \sqrt{\frac{\rho \omega}{2 \pi \eta_{p}}}, R e=\frac{\rho W_{0} R_{0}}{\eta_{p}} \text {, et } \varepsilon=\frac{R_{0}}{L}
$$

qui sont respectivement le nombre de Womersley, le nombre de Reynolds de l'écoulement et $\varepsilon$ un paramètre géométrique caractérisant la courbure de la paroi.

En microcirculation les faibles valeurs des rayons des conduits conduisent à des écoulements unidimensionnels [16]:

$\varepsilon^{2} \ll 1$ et $\operatorname{Re} \varepsilon \ll 1$.

Les effets d'inertie couplés aux effets de courbure sont négligés et il n'y a pas de gradient transversal de pression.

Dans le cadre de ces approximations, le système des équations locales, adimensionnelles, traduisant la conservation de la quantité de mouvement et de la masse s'écrit :

$$
\begin{aligned}
& -\frac{\partial P}{\partial r}=0 \\
& -\frac{\partial P}{\partial z}+\frac{1}{r} \frac{\partial}{\partial r}\left|m \frac{\partial W}{\partial r}\right|=\beta^{2} \frac{\partial W}{\partial t} \\
& \frac{\partial W}{\partial z}+\frac{1}{r} \frac{\partial}{\partial r}(r U)=0
\end{aligned}
$$

où

$$
\eta=\eta_{m}, W=W_{m} \text { et } U=U_{m} \text { dans le noyau } 0 \leq r \leq A
$$

avec

$$
\eta_{m}=K\left|\frac{\partial W_{m}}{\partial r}\right|^{n-1} \text { et } K=\frac{K^{*}}{\eta_{p}}\left[\frac{W_{0}}{R_{0}}\right]^{n-1} \text {. }
$$

et $\eta=1, W=W_{p}$ et $U=U_{p}$ dans le manchon périphérique $A \leq r \leq R$

L'indice $m$ (respectivement $p$ ) indique les grandeurs relatives au noyau (respectivement au manchon).

Les conditions aux limites, associées à ce système d'équations, qui traduisent la symétrie de l'écoulement et l'adhérence des particules fluides à la paroi sont :

$$
\begin{aligned}
& \frac{\partial W_{m}}{\partial r}=0, U_{m}=0 \text { pour } r=0 \\
& W_{p}=0, U_{p}=\frac{\beta^{2}}{\operatorname{Re\varepsilon } \frac{\partial R}{\partial t}} \\
& \text { pour } r=R .
\end{aligned}
$$


A l'interface, on impose la continuité du champ de vitesses et des contraintes tangentielles :

$$
W_{m}=W_{p}, U_{m}=U_{p} \text { et } \eta_{m} \frac{\partial W_{m}}{\partial r}=\frac{\partial W_{p}}{\partial r} \text { pour } r=A
$$

La loi du tube sous forme adimensionnelle s'écrit :

$$
\lambda \frac{\partial R^{2}}{\partial t}+R^{2}-1=a\left(P-P_{e x t}\right)
$$

où $S=\frac{S^{*}}{\pi R_{0}^{2}}=R^{2}, \lambda=\frac{\omega}{2 \pi} \lambda^{*}$ et $a=\frac{\eta_{p} L W_{0}}{\pi R_{0}} a^{*}$

Le gradient de pression étant inconnu le long du conduit, le système des équations locales ne peut être résolu séparément. On lui adjoint le système des équations intégrales de l'écoulement.

En intégrant les équations locales, chacune sur son domaine correspondant, le long d'une section, on obtient, en utilisant les règles d'intégration de Leibnitz et les conditions aux limites, deux équations qui sommées conduisent, en tenant compte des conditions de continuité, au système des équations intégrales :

$$
\begin{aligned}
& \frac{\partial Q}{\partial z}+\frac{\beta^{2}}{R e \varepsilon} \frac{\partial R^{2}}{\partial t}=0 \\
& \beta^{2} \frac{\partial Q}{\partial t}=-R^{2} \frac{\partial P}{\partial z}+2 R \tau_{p}
\end{aligned}
$$

où $Q$ est le débit global à travers la section $z$ du tube à l'instant $t$ et $\tau_{p}=\frac{\partial W_{p}}{\partial r}(r=R)$ la contrainte de cisaillement pariétale.

\section{IV — MÉTHODES ET PROCESSUS DE RÉSOLUTION}

L'ensemble des systèmes d'équations intégrales et locales est résolu à l'aide d'un schéma aux différences finies implicite [17].

Les calculs sont amorcés avec la valeur de la contrainte de cisaillement pariétale $\tau_{p}$ issue du profil initial, qui peut être absolument quelconque pourvu qu'il satisfasse aux

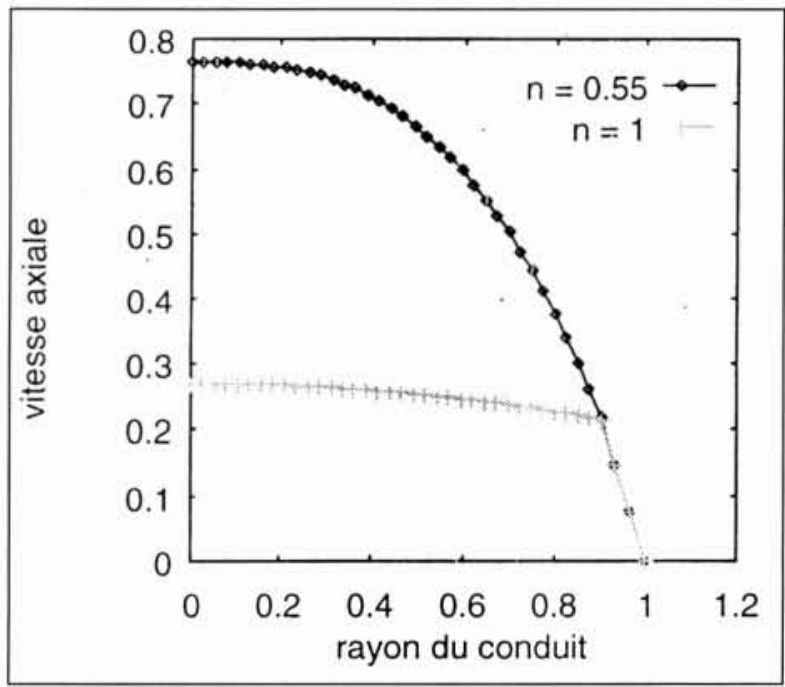

1. Evolution des profils des vitesses axiales en $Z=L / 2$ à $t=0$ pour deux valeurs de $n$. conditions aux limites. On choisit un profil qui soit assez proche du profil réel, on prendra dans ce but le profil correspondant à un écoulement de fluide à deux phases newtonienostwaldien en conduite axisymétrique rigide.

La résolution du système des équations intégrales permet, à chaque instant, la détermination de la section et du débit. On détermine au même instant à l'aide de la loi du tube la pression et le gradient de pression au point de l'écoulement considéré.

Les profils des vitesses à chaque instant peuvent être alors obtenus par la résolution des équations locales à partir desquelles, la grandeur $\tau$ est réestimée, sa valeur corrigée permet de réitérer et ce jusqu'à convergence de la solution.

Les critères de convergences et de périodicité de la solution portent sur la pression. Si $N$ est le nombre de cycles de calcul, on impose :

$$
\operatorname{Sup}\left|\frac{P_{N}(z, t)-P_{N+1}(z, t)}{P_{N}(z, t)}\right|<\varepsilon_{1}
$$

Le calcul doit être répété sur plusieurs périodes pour établir la périodicité de la solution. On a testé cette périodicité en imposant :

$$
\operatorname{Sup}\left|\frac{P_{N}(z, t)-P_{N}(z, t+1)}{P_{N}(z, t+1)}\right|<\varepsilon_{2}
$$

$\varepsilon_{1}$ et $\varepsilon_{2}$ étant deux quantités petites fixées à l'avance.

Les données du problème sont exprimées en termes de pression à l'entrée et à la sortie du conduit et la périodicité de la solution tient lieu de condition initiale.

\section{V — RÉSULTATS ET DISCUSSIONS}

Les figures 1, 2, 3 et 4 montrent les profils des vitesses axiales de l'écoulement diphasique newtonien-ostwaldien en comparaison avec le cas binewtonien aux instants $t=0$, $t=T / 4, t=T / 2$ et $t=3 T / 4$. Il y a diminution progressive des vitesses axiales en tout point et l'on peut prévoir un minimum en $t=T / 2$, puis l'amorce d'une augmentation progressive jusqu'à $t=T$.

On constate, comme l'ont déjà fait certains auteurs [3], que les profils de vitesses sont en phase avec le profil de pression (fig. 5), ce qui indique bien que les forces de viscosité sont prépondérantes par rapport aux forces d'inertie.

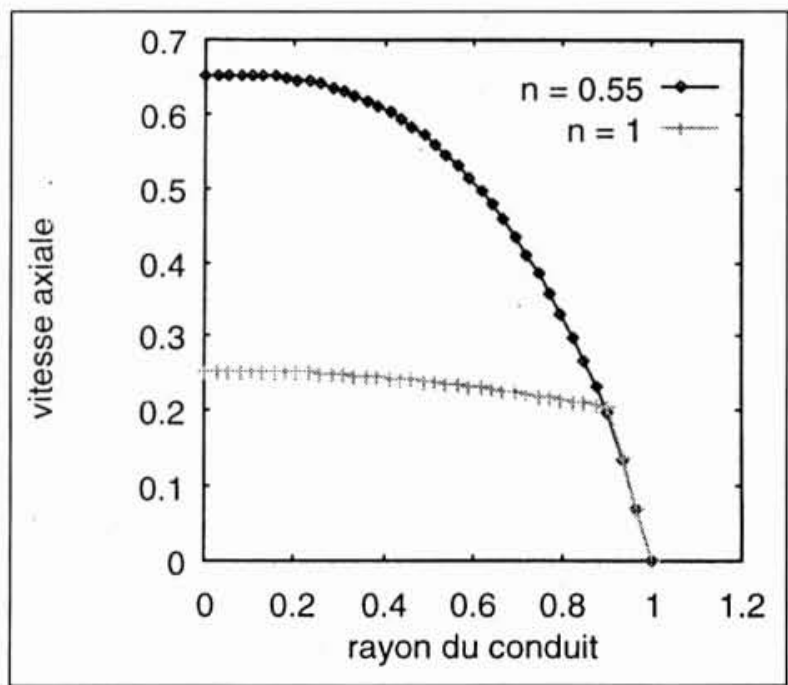

2. Evolution des profils des vitesses axiales en $Z=L / 2$ à $t=T / 4$ pour deux valeurs de $n$. 


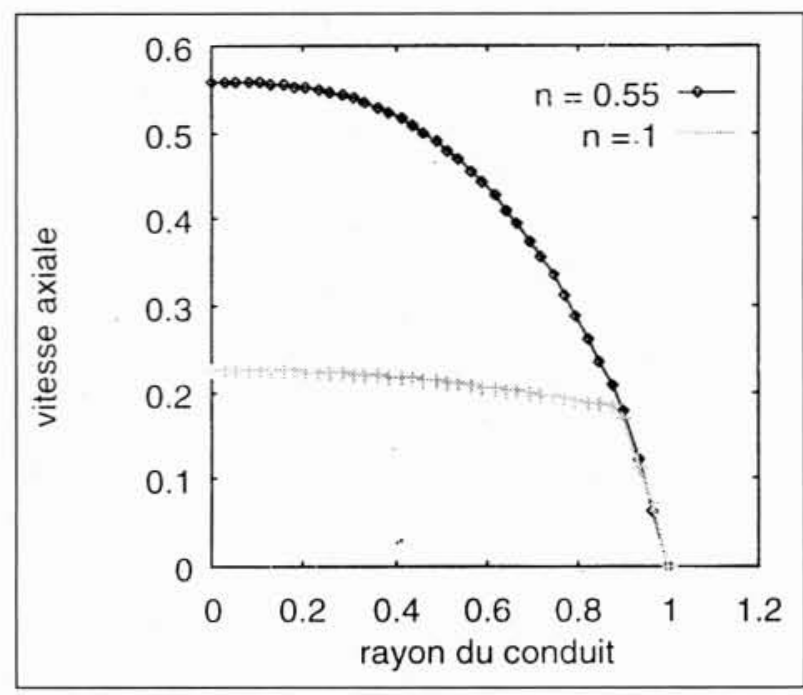

3. Evolution des profils des vitesses axiales en $Z=L / 2$ à $t$ $=T / 2$ pour deux valeurs de $n$.

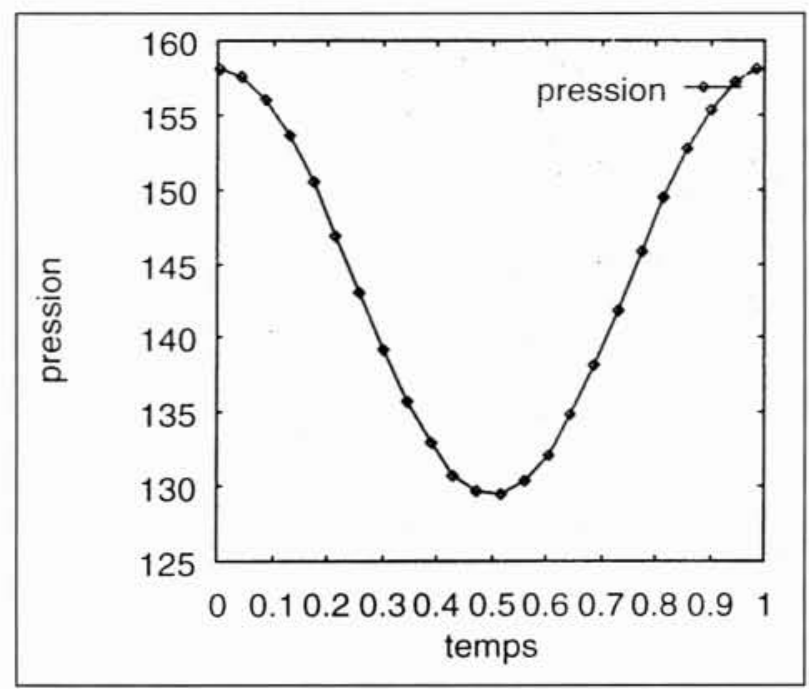

5. Evolution du profil de la pression en $z=L / 2$.

La cassure des profils correspondant à $n=1$, au point $r=0,9$ qui n'est autre que l'interface noyau-plasma, est due aux deux viscosités différentes des deux phases fluides. De ce fait en tenant compte de la condition de continuité des contraintes, les gradients de vitesses sont différents, d'où la différence entre les tangentes de la courbe du noyau et celle du plasma.

Sur chacune des figures 1, 2, 3 et 4 sont aussi portées les variations des profils des vitesses axiales en fonction de l'indice de comportement $n$, aux instants $(t=0, t=T / 4$, $t=T / 2$ et $t=3 T / 4)$. On constate qu'une diminution de $n$, qui se traduit par une pseudoplasticité plus marquée, s'accompagne d'une diminution de la viscosité apparente qui entraîne donc une augmentation des amplitudes des vitesses et ce pour une même valeur de la consistance $K$. L'augmentation de l'indice de comportement entraîne une augmentation de la viscosité et par la suite une augmentation des frottements des couches d'où le ralentissement de l'écoulement qui est à l'origine de l'aplatissement des profils des vitesses correspondant à $n=1$.

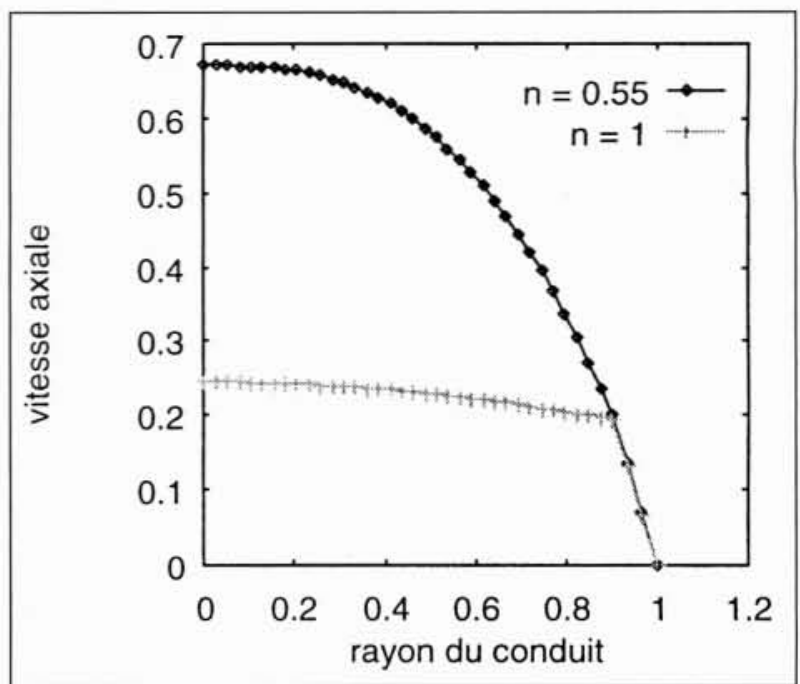

4. Evolution des profils des vitesses axiales en $Z=L / 2$ à $t$ $=3 T / 4$ pour deux valeurs de $n$.

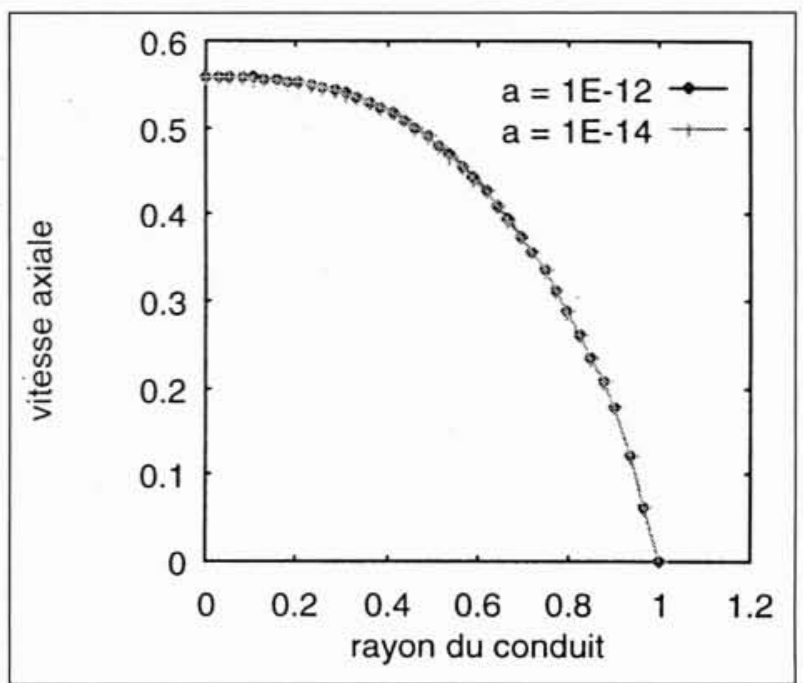

6. Evolution des profils des vitesses axiales en $z=L / 2$ à $t=T / 2$ pour deux valeurs de la compliance $a$.

La figure 6 montre l'évolution des profils des vitesses axiales pour deux valeurs de la compliance $a$. Ce paramètre n'a aucune influence sur ces profils, ce qui est dû au fait que les valeurs prises par $a$ sont très faibles et ne peuvent avoir d'influence notoire sur l'écoulement. Ces valeurs correspondent aux valeurs de la compliance au niveau de la microcirculation sanguine.

La figure 7 permet de juger de l'importance du temps de relaxation $\lambda$ sur l'écoulement, on a effectué le calcul pour deux valeurs $(0,001$ et 0,4$)$. Une augmentation du temps de relaxation $\lambda$ se traduit par un accroissement des valeurs des vitesses axiales.

La figure 8 indique les variations des profils des vitesses axiales en fonction de la consistance $K$, du fluide. L'influence de ce paramètre rhéologique est à rapprocher de celle de la viscosité dans le cas newtonien. Les variations de $K$ influent sur les profils des vitesses axiales et ce pour un même indice de comportement. Il y a diminution des valeurs des vitesses axiales et aplatissement plus au moins marqué des profils au niveau du noyau. Cela est dû à l'accroissement du frottement interne entre les diverses couches coaxiales du fluide lorsque $K$ augmente. 


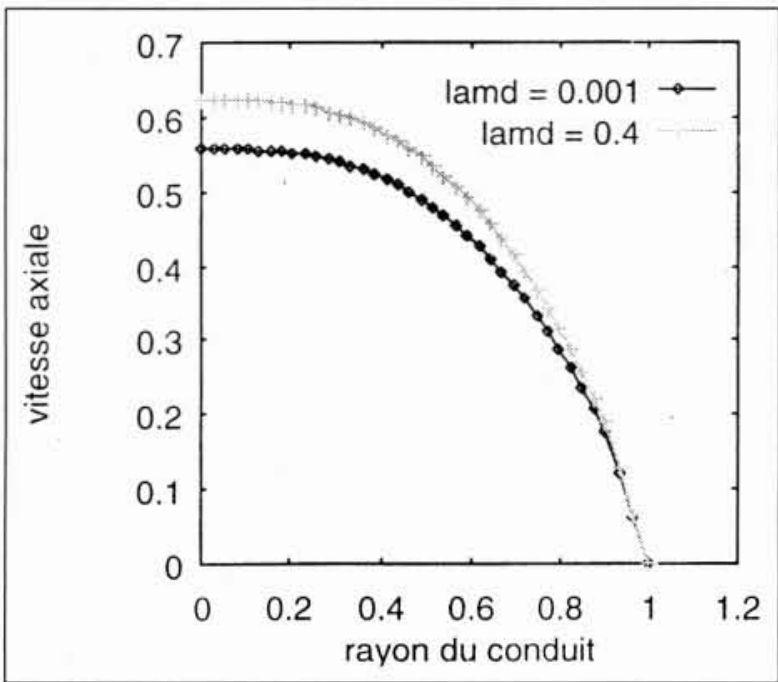

7. Evolution des profils des vitesses axiales en $z=L / 2$ à $t=T / 2$ pour deux valeurs de temps de relaxation.

\section{VI $\square$ CONCLUSION}

Dans cette étude, qui a traité des écoulements pulsés de fluides diphasiques, couche plasmatique newtonienne et noyau central obéissant à la loi d'Ostwald, nous avons montré l'importance de certains paramètres rhéologiques aussi bien du fluide, que de la paroi. En effet, les profils des vitesses axiales montrent l'importance de l'indice de comportement $n$ et de la consistance $K$ du fluide ainsi que I'influence du temps caractéristique $\lambda$ caractérisant la viscoélasticité de la paroi. L'écoulement est pratiquement gouverné par ces trois paramètres.

\section{LISTE DES SYMBOLES}

$L \quad$ longueur du conduit

$R_{0} \quad$ rayon au repos du conduit

$r$ et $z \quad$ coordonnées cylindriques en symétrie de révolution

$t \quad$ temps

$R(z, t) \quad$ rayon du conduit à l'état déformé

$\lambda$ temps de relaxation lié à la viscoélasticité de la paroi

$S(z, t) \quad$ section à l'instant $t$

$S_{0} \quad$ section à l'état de repos

$P \quad$ pression à l'intérieur du conduit

$P_{\mathrm{eut}} \quad$ pression à l'extérieur du conduit

a compliance de la paroi

$W(r, z, t)$ vitesse axiale de l'écoulement

$U(r, z, t)$ vitesse radiale de l'écoulement

$A(z, t) \quad$ rayon de la phase centrale (noyau)

$\eta_{m} \quad$ viscosité du noyau

$K^{m} \quad$ consistance du noyau

$n \quad$ indice de comportement du fluide constituant le noyau

$\eta_{p} \quad$ viscosité de la couche plasmatique

d $^{p} \quad$ tenseur des taux de déformations

$\alpha \quad$ épaisseur de la couche plasmatique

$\rho \quad$ masse volumique commune aux deux fluides

$\Sigma \quad$ tenseur des contraintes

1 tenseur unité

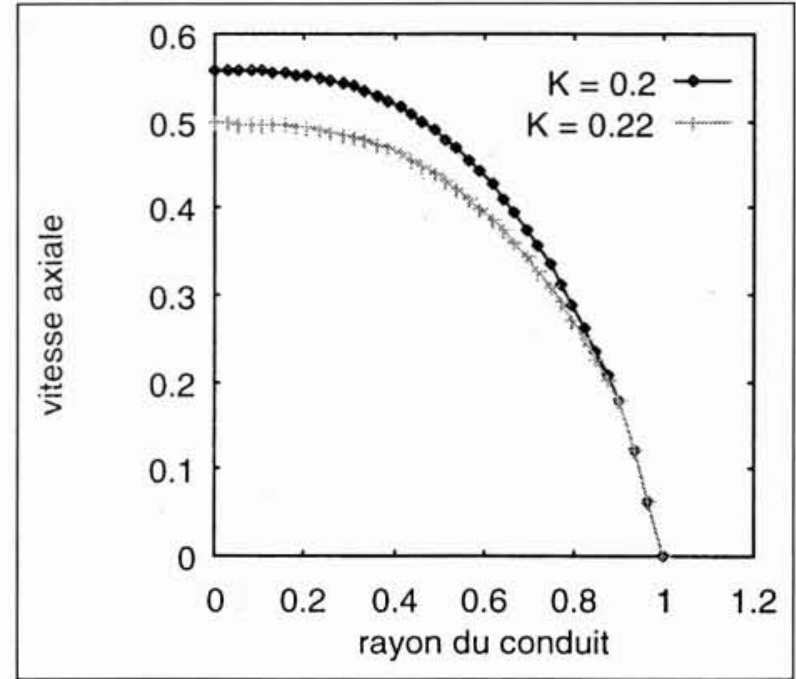

8. Evolution des profils de vitesses axiales en $z=L / 2$ à $t=T / 2$ pour deux valeurs de la consistance $K$ pour un même indice $n$.

$V(r, z, t)$ champ de vitesses

$\omega$ pulsation du phénomène

$W_{0} \quad$ vitesse caractéristique de l'écoulement dans la direction axiale

$\beta \quad$ nombre de Womersley

Re nombre de Reynolds

$\varepsilon \quad$ paramètre de forme caractérisant la courbure de la paroi

$Q(z, t)$ débit global à travers la section $z$ du tube à l'instant $t$

$\tau_{p} \quad$ contrainte de cisaillement pariétale

\section{RÉFÉRENCES}

[1] Chaturani P and Upadhya V.S. - A two-fluid model for blood flow through small diameter tubes. Biorrheology Vol. 16, pp. 109-118, 1978.

[2] BıтоuN J.-P. - Etudes théorique et expérimentale de la microcirculation sanguine au passage d'une sténose. Thèse de doctorat, I.N.P., Toulouse, 1985.

[3] ZegGwaGH G. - Modélisations théoriques et expérimentales de l'hémodynamique en microcirculation. Thèse de Doctorat ès-Sciences, I.N.P., Toulouse, 1988.

[4] Kerroum M. - Ecoulements pulsés de fluides diphasiques en conduites déformables de faibles diamètres. Modélisation de la microcirculation sanguine. Thèse de Doctorat ès-Sciences, Rabat. 1994.

[5] THEODOROu G. - Ecoulements non permanents de fluides non newtoniens dans un rétrécissement de section. Thèse de Docteur ingénieur, I.N.P. Toulouse, 1983.

[6] TAZı M. - Modélisation d'écoulements sanguins au passage de bifurcation. Thèse de doctorat, I.N.P., Toulouse, 1991.

[7] Chaturani P and Kalani P.N. - Two layered Poiseuille flow model for blood flow through arteries of small diameter and arterioles. Biorheology 13, 243-250, 1976.

[8] Bugliarello G. and Sevilla J. - Velocity distribution and other characteristics of steady and pulsatile blood flow in fine glass tubes. Biorheology 7, 85,107, 1970. 
[9] Bugliarello G., Kapur C and Hsiao G. - In : Symposium on Biorheology. A.L. Copley (Ed.) New York, London and sydney : interscience publ. 1965.

[10] Popel A.S., Regirer S.A. and Usick P.I. - A continum model of blood flows. Biorheology 11, 427-437, 1974.

[11] HAYNES H.R. - Physical basis of the dependence of blood viscosity on tube radius. Am. J. Physiol 198(6), 11932000,1960 .

[12] Goldsmith H.L. and SKalaK R. - Hemodynamics. In : annual Review of fluid Dynamics. M. Van Dyke (Ed.) California : Annual Review Inc. Palo Alto Publ. pp. 231247, 1975.

[13] Buthaud H. - Analyse non-newtonienne de l'écoulement sanguin dans un modèle de l'aorte. Thèse de docteur ingénieur., Université de Poitiers, 1977.

[14] Rakotomalala A.R. and Bellet D. - Ecoulements transitoires et périodiques de fluides non newtoniens en conduites tronconiques, Journal de Physique I(1), 87-102, 1991.
[15] SKalak T.C., sCHMID-sCHONBEIN G.W. - Viscoelastic properties of microvessels in rat spinotrazius muscle. J. Biom. Eng., Vol. 108, pp. 93-200, 1986.

[16] Burton A.G. - Physiologie et biophysique de la circulation. Masson, Paris, 1967.

[17] Novgier J.-P. - Méthodes de calcul numérique. $3^{e}$ Ed., Masson, Paris, 1989.

[18] Poiseuille J.M. - Recherches expérimentales sur le mouvement des liquides dans les tubes de très petits diamètres. Comptes rendus hebdomadaires des séances de l'académie des Sciences, 11, 961-967, 1041-48, 1840.

[19] Fahreus R. - The suspension stability of the blood. Physiological Revieus, Vol. IX n’ 2 april, 1929.

[20] Fahreus R., Lindqvist T. - The viscosity of the blood in narrow capillary tube. Am. J. Physiol. 96, 562 (1931).

[21] Middleman S. - Transport phenomena in the cardiovascular system. Wiley-Interscience, London, 1972.

[22] Comolet R. - Biomécanique circulatoire. Masson, Paris, 1984. 